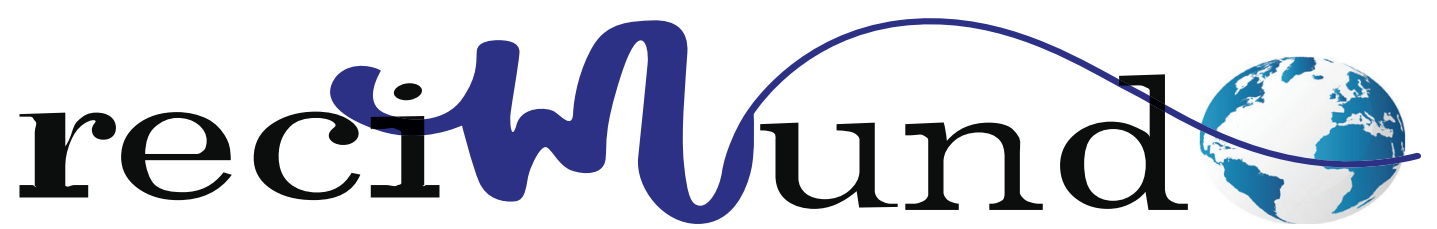

Revista Científica Mundo de la Investigación y el Conocimiento

DOI: 10.26820/recimundo/5.(1).enero.2021.120-128

URL: http://recimundo.com/index.php/es/article/view/991

EDITORIAL: Saberes del Conocimiento

REVISTA: RECIMUNDO

ISSN: 2588-073X

TIPO DE INVESTIGACIÓN: Artículo de Revisión

CÓdigo UNESCO: 32 Ciencias Médicas

PAGINAS: $120-128$

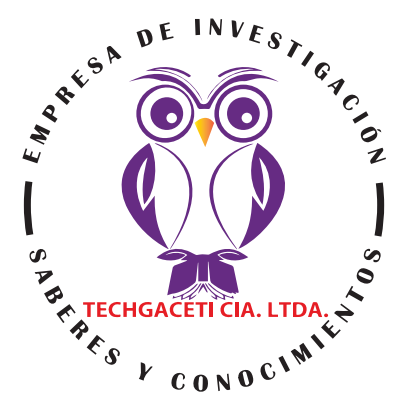

\title{
Relación del síndrome metabólico en el riesgo de enfermedades cardiovasculares
}

Relationship of the metabolic syndrome to cardiovascular disease risk Relação da síndrome metabólica com o risco de doenças cardiovasculares Carla María Araujo Egas'; Karla Misshell Castillo Aldás; Juan Carlos Londoño Mateus; Karen Michel Revelo Benavides ${ }^{4}$

RECIBIDO: 28/11/2020 ACEPTADO: 06/12/2020 PUBLICADO: 31/01/2021

1. Médico Cirujano de la Universidad UTE; Médico Rural / Centro de Salud Curipungo, Sangolqui, Ecuador; carlmari94@ hotmail.com; (D) https://orcid.org/0000-0003-4992-0724

2. Médico Cirujano de la Universidad Regional Autónoma de Los Andes "Uniandes"; Médico Rural Unidad Operativa Santa Bárbara; Sucumbíos, Ecuador; karlamisshell67678@gmail.com; (iD https://orcid.org/0000-0001-7544-9337

3. Médico Cirujano de la Universidad de Las Américas; Medico Rural / Centro de Salud Luxemburgo Ministerio de Salud Pública; Macas, Ecuador; juancaz17@hotmail.com; (iD https://orcid.org/0000-0002-5177-6007

4. Médico Cirujano de la Universidad de Las Américas; Médico Rural / Centro de Salud de Tufiño; Tulcán, Ecuador; karito_revelo_1995@hotmail.com; (D) https://orcid.org/0000-0002-5064-0116

CORRESPONDENCIA

Carla María Araujo Egas

carlmari94@hotmail.com

Quito, Ecuador

() RECIMUNDO; Editorial Saberes del Conocimiento, 2021 


\section{RESUMEN}

El síndrome metabólico (MetS) se ha definido de diferentes formas, sin embargo, los componentes claves comunes a la mayoría de las definiciones son los diferentes factores de riesgo que incluyen adiposidad abdominal, alteración de la glucosa en ayunas, hipertensión y dislipidemia. Un mediador principal del MetS parece ser la resistencia a la insulina, que se relaciona con el desarrollo de disfunciones vasculares y metabólicas que preceden a la enfermedad cardiovascular manifiesta y la diabetes tipo 2. La evidencia sugiere que los mecanismos subyacentes al elevado riesgo cardiovascular asociado con el MetS comienzan con un daño orgánico subclínico. La terapia para MetS se dirige a componentes individuales del síndrome e incluye intervenciones en el estilo de vida, terapia modificadora de lípidos y agentes antihipertensivos, en particular aquellos que inhiben el sistema renina-angiotensina. Los resultados de los ensayos de inhibidores de la enzima convertidora de angiotensina y bloqueadores de los receptores de angiotensina han demostrado reducciones en la diabetes de nueva aparición y eventos cardiovasculares en una amplia gama de pacientes. Actualmente se están llevando a cabo ensayos clínicos para investigar más el papel de estos fármacos en la prevención primaria de la diabetes tipo 2 en pacientes con MetS. El propósito de este artículo es revisar el MetS desde la perspectiva cardiológica con la esperanza de que una mejor comprensión de los vínculos entre el MetS y la enfermedad cardiovascular pueda conducir a una mejor gestión de las personas en riesgo.

Palabras clave: Sistema metabólico, enfermedad cardiovascular, resistencia a la insulina, diabetes tipo 2.

\section{ABSTRACT}

Metabolic syndrome (MetS) has been defined in different ways, however, the key components common to most definitions are different risk factors including abdominal adiposity, impaired fasting glucose, hypertension, and dyslipidemia. A major mediator of MetS appears to be insulin resistance, which is associated with the development of vascular and metabolic dysfunctions that precede overt cardiovascular disease and type 2 diabetes. Evidence suggests that the mechanisms underlying the elevated cardiovascular risk associated with MetS begins with subclinical organ damage. Therapy for MetS targets individual components of the syndrome and includes lifestyle interventions, lipid modifying therapy, and antihypertensive agents, particularly those that inhibit the renin-angiotensin system. Results from trials of angiotensin converting enzyme inhibitors and angiotensin receptor blockers have shown reductions in new-onset diabetes and cardiovascular events in a wide range of patients. Clinical trials are currently underway to further investigate the role of these drugs in primary prevention of type 2 diabetes in MetS patients. The purpose of this article is to review MetS from a cardiological perspective in the hope that a better understanding of the links between MetS and cardiovascular disease may lead to better management of people at risk.

Keywords: Metabolic system, cardiovascular disease, insulin resistance, type 2 diabetes.

\section{RESUMO}

A síndrome metabólica (MetS) tem sido definida de diferentes maneiras, no entanto, os principais componentes comuns à maioria das definições são diferentes fatores de risco, incluindo adiposidade abdominal, glicose de jejum prejudicada, hipertensão e dislipidemia. Um dos principais mediadores da SM parece ser a resistência à insulina, que está associada ao desenvolvimento de disfunções vasculares e metabólicas que precedem as doenças cardiovasculares evidentes e o diabetes tipo 2. As evidências sugerem que os mecanismos subjacentes ao elevado risco cardiovascular associado à SM começam com a lesão de órgãos subclínicos. A terapia para MetS visa componentes individuais da síndrome e inclui intervenções no estilo de vida, terapia modificadora de lipídios e agentes anti-hipertensivos, particularmente aqueles que inibem o sistema renina-angiotensina. Os resultados de ensaios com inibidores de enzimas conversoras de angiotensina e bloqueadores de receptores de angiotensina mostraram reduções no diabetes recém-estabelecido e eventos cardiovasculares em uma ampla gama de pacientes. Atualmente, estão sendo realizados ensaios clínicos para investigar melhor o papel desses medicamentos na prevenção primária do diabetes tipo 2 em pacientes com MetS. O objetivo deste artigo é rever a MetS de uma perspectiva cardiológica, na esperança de que uma melhor compreensão das ligações entre a MetS e a doença cardiovascular possa levar a um melhor gerenciamento de pessoas em risco.

Palavras-chave: Sistema metabólico, doença cardiovascular, resistência à insulina, diabetes tipo 2. 


\section{Introducción}

La agrupación dentro de un individuo de ciertos factores de riesgo de enfermedad cardiovascular (ECV), como obesidad abdominal, alteración de la glucosa en ayunas, dislipidemia e hipertensión, se denomina MetS. Este conjunto de factores está asociado con un mayor riesgo de resultados adversos que exceden el riesgo asociado con las mismas condiciones consideradas por separado. El MetS y sus consecuencias presentan un desafío para el sistema de salud, particularmente en vista de la creciente prevalencia de sobrepeso / obesidad y diabetes mellitus tipo 2. Sin embargo, la explicación en curso de los mecanismos fisiopatológicos del MetS también proporciona nuevos objetivos para el manejo y una oportunidad para disminuir la carga de enfermedad asociada con estas condiciones.

El síndrome de resistencia a la insulina descrito inicialmente por Reaven en 1988, fue redefinido como "síndrome metabólico (MetS) por el informe del Panel III de Tratamiento de Adultos del Programa Nacional de Educación sobre el Colesterol, y ahora se acepta ampliamente como un problema de salud, situación que aumenta el riesgo de enfermedad cardiovascular (ECV)" (Zimmet, Alberti, \& Shaw, 2001). El MetS es un conjunto de condiciones asociadas como obesidad, presión arterial elevada (PA), tolerancia alterada a la glucosa (IGT), resistencia a la insulina, triglicéridos (TG) elevados y concentraciones bajas de colesterol de lipoproteínas de alta densidad (HDL).

Cada una de las afecciones asociadas tiene un efecto independiente, pero al agruparse se vuelven sinérgicas, lo que aumenta el riesgo de desarrollar ECV. "Por ejemplo, el gran estudio internacional INTERHEART mostró relaciones lineales entre estos factores de riesgo y el infarto de miocardio (IM)" (Rizza, Vigersky, \& Rodbard, 2003).
Las definiciones de MetS son confusas. Desde hace años, varios investigadores y organizaciones han sugerido diferentes definiciones, no obstante, todos coinciden en que las características del MetS incluyen dislipidemia aterogénica, estado protrombótico, resistencia a la insulina, hipertensión, obesidad abdominal, así como microalbuminuria elevada, fibrinógeno elevado, activador 1 del plasminógeno disminuido (PA-1), inhibidor del activador del plasminógeno elevado -1 (PAI-1), aumento de la viscosidad de la sangre y aumento del ácido úrico. (Rizza, Vigersky, \& Rodbard, 2003)

Cada anomalía promueve la aterosclerosis de forma independiente, pero cuando se agrupan, estos trastornos metabólicos son cada vez más aterogénicos y aumentan el riesgo de morbilidad y mortalidad cardiovascular. "Además, el MetS se ha asociado con el desarrollo de enfermedad coronaria (CHD), accidente cerebrovascular, diabetes mellitus tipo 2 (DM2), nefropatía diabética, retinopatía y neuropatía distal" (Kahn, Buse, Ferrannini, \& Stern, 2005).

La Declaración Interina Conjunta del Grupo de Trabajo sobre Epidemiología y Prevención de la Federación Internacional de Diabetes destacó que "no debería haber un componente obligatorio para el MetS, sino que todos los componentes individuales deberían considerarse importantes en la predicción del riesgo de ECV" (Kahn, Buse, Ferrannini, \& Stern, 2005). Los criterios para diagnosticar el SM según el informe del panel de tratamiento de adultos III (ATP III) del Programa Nacional de Educación sobre el Colesterol son los siguientes: 1) obesidad abdominal, expresada como circunferencia de cintura, hombres $>102 \mathrm{~cm}$ y mujeres $>88$ $\mathrm{cm}, 2)$ glucosa en ayunas $110 \mathrm{mg} / \mathrm{dl}, 3)$ PA 130/80 mmHg, 4) TG 150 mg / dl.

A pesar de que los programas de educación pública instan a adultos y niños a modificar sus estilos de vida para incluir opciones de alimentos saludables y más actividad físi- 
ca, la mayoría de las personas no siguen las pautas dietéticas o de ejercicio recomendadas para mantener la salud. De esta manera surge la necesidad de realizar un estudio bibliográfico, donde se exponga la incidencia que tiene el síndrome metabólico sobre las enfermedades cardiovasculares con aras de brindar información de interés que sirva para futuras investigaciones.

\section{Metodología}

Para el desarrollo de este proceso investigativo, se plantea como metodología la encaminada hacia una orientación científica particular que se encuentra determinada por la necesidad de indagar en forma precisa y coherente una situación, en tal sentido Davila, (2015) define la metodología "como aquellos pasos anteriores que son seleccionados por el investigador para lograr resultados favorables que le ayuden a plantear nuevas ideas" (p.66)

Lo citado por el autor, lleva a entender que el desarrollo de la acción investigativa busca simplemente coordinar acciones enmarcadas en una revisión bibliográfica con el fin de complementar ideas previas relacionadas Relación del síndrome metabólico en el riesgo de enfermedades cardiovasculares a través de una revisión de literatura, para así finalmente elaborar un cuerpo de consideraciones generales que ayuden a ampliar el interés propuesto.

\section{Tipo de Investigación}

Dentro de toda práctica investigativa, se precisan acciones de carácter metodológico mediante las cuales se logra conocer y proyectar los eventos posibles que la determinan. En este sentido, la presente investigación corresponde al tipo documental, definido por Castro (2016), "se ocupa del estudio de problemas planteados a nivel teórico, la información requerida para abordarlos se encuentra básicamente en materiales impresos, audiovisuales y / o electrónicos". (p.41).
En consideración a esta definición, la orientación metodológica incluye la oportunidad de cumplir con una serie de actividades inherentes a la revisión y lectura de diversos documentos, donde se encuentran ideas explicitas relacionadas con los tópicos encargados de identificar una característica inmersa en el estudio. Por lo tanto, se realizaron continuas interpretaciones con el claro propósito de revisar aquellas apreciaciones propuestas por diferentes investigadores en relación al tema de interés, para luego dar la respectiva argumentación a los planteamientos, en función a las necesidades encontradas en la investigación, apoyados en las herramientas tecnológicas para la búsqueda de trabajos con valor científico disponibles en la web que tenían conexión con el objetivo principal de la investigación.

\section{Fuentes Documentales}

El análisis correspondiente a las características que predomina en el tema seleccionado, llevan a incluir diferentes fuentes documentales encargadas de darle el respectivo valor científico y en ese sentido cumplir con la valoración de los hechos a fin de generar nuevos criterios que sirven de referencia a otros procesos investigativos. Para Castro,(2016) las fuentes documentales incorporadas en la investigación documental o bibliográfica, "representa la suma de materiales sistemáticos que son revisados en forma rigurosa y profunda para llegar a un análisis del fenómeno" (p.41). Por lo tanto, se procedió a cumplir con la lectura previa determinada para encontrar aquellos aspectos estrechamente vinculados con el tema, con el fin de explicar mediante un desarrollo las respectivas apreciaciones generales de importancia.

\section{Técnicas para la Recolección de la Infor- mación}

La conducción de la investigación para ser realizada en función a las particularidades que determinan a los estudios documenta- 
les, tiene como fin el desarrollo de un conjunto de acciones encargadas de llevar a la selección de técnicas estrechamente vinculadas con las características del estudio. Bolívar, (2015), refiere, que es "una técnica particular para aportar ayuda a los procedimientos de selección de las ideas primarias y secundarias". (p.71).

Tal como lo expresa, Bolívar, (2015) "Las técnicas documentales proporcionan las herramientas esenciales y determinantes para responder a los objetivos formulados y llegar a resultados efectivos" (p. 58). Es decir, para responder con eficiencia a las necesidades investigativas, se introdujeron como técnica de recolección el método inductivo, que hizo posible llevar a cabo una valoración de los hechos de forma particular para llegar a la explicación desde una visión general. El autor Bolívar, (2015) tambien expresa que las técnicas de procesamiento de datos en los estudios documentales "son las encargadas de ofrecer al investigador la visión o pasos que deben cumplir durante su ejercicio, cada una de ellas debe estar en correspondencia con el nivel a emplear" (p. 123). Esto indica, que para llevar a cabo el procesamiento de los datos obtenidos una vez aplicadas las técnicas seleccionadas, tales como: fichas de resumen, textual, registros descriptivos entre otros, los mismos se deben ajustar al nivel que ha sido seleccionado.

\section{Resultados}

La diabetes es un importante problema de salud pública en todo el mundo. La diabetes tipo 2 representa más del 90\% de todos los casos. La American Heart Association, (2005) expone que:

A nivel mundial, la diabetes afectó aproximadamente al $2,8 \%$ de todos los grupos de edad, o 171 millones de personas en 2000. Se espera que esta cifra aumente, con un aumento proyectado a
366 millones (4,4\%) afectados con diabetes en todo el mundo para el año 2030.

La diabetes no diagnosticada y las condiciones prediabetes, como la tolerancia a la glucosa alterada y la glucosa en ayunas alterada, son comunes. En la actualidad, existen muy pocos endocrinólogos en ejercicio que tratan a adultos en comparación con los millones de personas con diabetes diagnosticada, diabetes no diagnosticada y diabetes inminente en forma de MetS

En tal sentido, Rizza, Vigersky, \& Rodbard, (2003) afirman que "la tasa actual de endocrinólogos que se incorporan a la fuerza laboral, no dará abasto para evaluar, identificar y manejar a todas las personas que requieren cuidados metabólicos". Los cardiólogos y los médicos extensores cardiovasculares están en una posición ideal no solo para identificar a los pacientes con MetS, sino también para proporcionar un manejo más agresivo de los factores de riesgo (en particular, dislipidemia e hipertensión) dirigidos a retrasar o prevenir el desarrollo de la diabetes.

\section{¿Qué es el síndrome metabólico?}

Aunque recientemente se ha cuestionado la designación del MetS como una condición fisiopatológica única y como un predictor de enfermedad, la mayoría de los médicos e investigadores han sostenido durante mucho tiempo que ciertos factores de riesgo metabólico son propensos a agruparse, y que esta agrupación aumenta el riesgo de ECV y diabetes. "Se ha propuesto una variedad de definiciones para MetS, todas incluyen componentes de obesidad, dislipidemia, hipertensión y niveles elevados de glucosa en ayunas, pero difieren en cuántos y cuáles de estos componentes son esenciales para el diagnóstico" (Alberti, Zimmet, \& Shaw, 2005). 
La resistencia a la insulina y la obesidad generalmente se consideran dos factores de riesgo subyacentes importantes para el MetS.

La resistencia a la insulina puede ser causada por el exceso de grasa corporal, en particular la obesidad abdominal, y por la inactividad física, y está asociada con un aumento de la presión arterial a través de varios mecanismos diferentes. La resistencia crónica a la insulina conduce a intolerancia a la glucosa y diabetes tipo 2 , que es un factor de riesgo independiente de ECV (Alberti, Zimmet, \& Shaw, 2005).

La obesidad abdominal se puede evaluar de diversas formas; varios conjuntos diferentes de criterios ahora especifican que la circunferencia de la cintura se evalúe utilizando valores específicos para diferentes grupos étnicos / raciales.

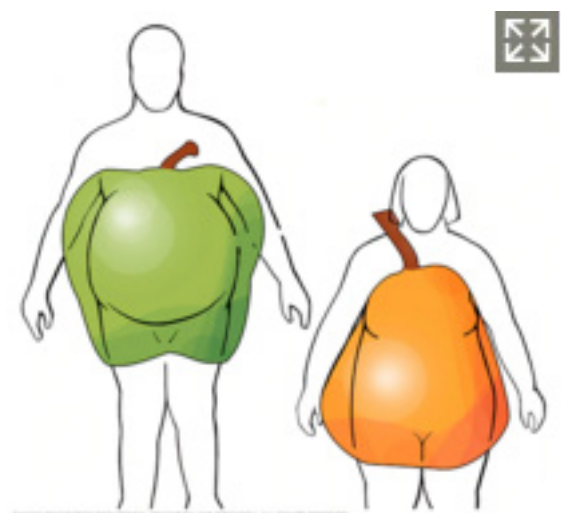

Figura 1. Cuerpos con forma de manzana y de pera

Fuente: (Mayo Clinic, 2019)

\section{Resistencia a la insulina y enfermedad vascular}

Los mecanismos que subyacen al riesgo elevado de ECV asociado con el MetS parecen implicar daño subclínico de órganos diana. Entre los pacientes con hipertensión pero sin diabetes, los que tienen MetS "parecen tener más probabilidades de tener una mayor prevalencia de microalbuminu- ria e hipertrofia ventricular izquierda, mayor índice de masa ventricular izquierdo y aumento del grosor de la íntima carotídea que aquellos sin MetS" (Leoncini, Ratto, Viazzi, \& Vaccaro, 2015). Cuanto mayor es el número de componentes del MetS presentes, mayor es la microalbuminuria y el índice de masa del ventrículo izquierdo. Además, el MetS se asoció con un mayor riesgo de daño de órganos diana que cualquiera de sus componentes individuales.

Es probable que muchos mecanismos diferentes contribuyan a la fisiopatología subyacente a la asociación entre MetS y un mayor riesgo de eventos cardiovasculares ateroscleróticos. Los autores Prasad \& Quyyumi, (2014) exponen.

Las alteraciones en las vías metabólicas, las reacciones inflamatorias y otros procesos celulares pueden aumentar el riesgo de aterosclerosis en el estado de resistencia a la insulina. Por ejemplo, existe una creciente evidencia de interacciones celulares entre las vías de señalización involucradas en las acciones de la insulina y el sistema renina-angiotensina (RAS) (p. 507).

El RAS, a través de la angiotensina II, está involucrado no solo en la patogénesis de la hipertensión y la aterosclerosis, sino que también parece jugar un papel en el desarrollo de resistencia a la insulina. La obesidad también predispone a las enfermedades cardiovasculares: el tejido adiposo actúa como un órgano endocrino, secretando hormonas y otras sustancias que crean un estado proinflamatorio y promueven la formación de placas ateroscleróticas (Lau \& Dhillon, 2015).

Los receptores activados por proliferadores de peroxisomas nucleares (PPAR) también parecen desempeñar un papel en los mecanismos aterogénicos subyacentes al

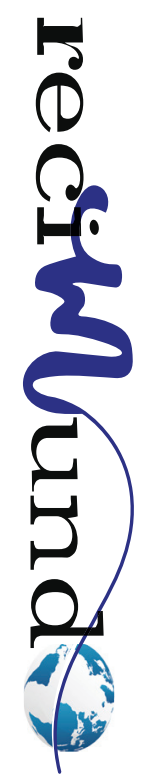


MetS. "Los PPAR regulan la expresión de una variedad de genes y modulan el metabolismo de los lípidos, el control glucémico y la inflamación y el tono vasculares" (Lau \& Dhillon, 2015). La modulación de las acciones relacionadas con los PPAR por factores de riesgo cardiovascular, como la obesidad y el sobrepeso, puede conducir a la promoción de la aterosclerótica.

\section{Enfoques terapéuticos}

Independientemente de la definición particular de MetS utilizada, es fundamental tratar cada factor de riesgo cardiovascular de forma agresiva. Este enfoque ciertamente se aplica a la alteración de la glucosa en ayunas, la dislipidemia y la hipertensión.

El Grupo de Investigación del Programa de Prevención de la Diabetes evaluó los beneficios de la intervención en el estilo de vida (pérdida de peso y aumento de la actividad física) en comparación con el agente antihiperglucémico metformina para la prevención de la diabetes en personas con concentraciones elevadas de glucosa en ayunas y poscarga (Knowler \& Barrett, 2012).

Ambos tratamientos disminuyeron la diabetes de nueva aparición, pero la intervención en el estilo de vida dio lugar a una incidencia de diabetes del 39\% (intervalo de confianza [IC] del (95\%, 24 - 51\%) menor que el tratamiento con metformina ( $p<0,001)$.

Los fármacos dirigidos a PPAR-alfa (p. Ej., Fenofibrato y gemfibrozil) y PPAR-gamma (p. Ej., Tiazolidindionas como rosiglitazona y pioglitazona) también "se utilizan en el tratamiento de MetS. Los fibratos disminuyen Ios triglicéridos, aumentan el colesterol de las lipoproteínas de alta densidad y pueden tener algunos efectos antiinflamatorios; sin embargo, se sigue evaluando su efecto sobre los resultados de las ECV" (Miranda \& DeFronzo, 2015).
Las tiazolidinedionas aumentan la sensibilidad a la insulina, aumentan la captación de glucosa del músculo esquelético y disminuyen los niveles plasmáticos de ácidos grasos libres, y se ha demostrado que reducen la progresión a diabetes en personas con niveles elevados de glucosa en ayunas. Adicionalmente, aumentan el colesterol de lipoproteínas de alta densidad, generalmente reducen los triglicéridos y reducen la inflamación, aunque su efecto sobre el colesterol de lipoproteínas de baja densidad puede variar según el fármaco (Knowler, Hamman, \& Edelstein, 2015).

Los agentes antihipertensivos tienen diferentes efectos sobre los componentes del MetS. Se sabe que los diuréticos y los betabloqueantes tienen efectos metabólicos que promueven condiciones favorables para el desarrollo de la diabetes, los autores Pepine \& Cooper-Dehoff, (2014) indican que.

Por el contrario, los pacientes sin diabetes tratados con regímenes (antihipertensivos) que contienen inhibidores de la enzima convertidora de angiotensina (ECA) y bloqueadores de los receptores de angiotensina (BRA) muestran un mejor metabolismo de la glucosa y una mayor sensibilidad a la insulina en comparación con los regímenes que contienen betabloqueantes y diuréticos (p. 512).

La Evaluación de Prevención de Resultados Cardíacos (HOPE) "informó que 5 años de tratamiento con ramipril en pacientes con enfermedad vascular redujeron significativamente la incidencia de diabetes de nueva aparición (3,6\% frente a 5,4\% con placebo; $p<0,001$ )" (Yusuf \& Sleight, 2010). En una población de pacientes hipertensos con enfermedad de las arterias coronarias, el International Verapamil SR TrandolaprilStudy (INVEST) "mostró tasas más bajas de diabetes recién diagnosticada en sujetos alea- 
torizados a la estrategia de verapamil SR, que fueron expuestos al inhibidor de la ECA trandolapril, en la estrategia de atenolol $(7,0$ y $8,2 \%$, respectivamente) durante 2,7 años de seguimiento" (Pepine \& Cooper-Dehoff, 2014). Sin embargo, los ensayos con ARA II han mostrado resultados similares.

\section{Conclusión}

La creciente prevalencia de la diabetes tipo 2 y la continua alta tasa de morbilidad y mortalidad cardiovascular subrayan la importancia del reconocimiento y diagnóstico de los factores de riesgo metabólico asociados. El daño vascular y de órganos diana asociado con la resistencia a la insulina y otros componentes de lo que se ha denominado MetS comienza mucho antes de que la enfermedad cardiovascular o la diabetes tipo 2 se vuelva clínicamente evidente. Los cardiólogos están posicionados para desempeñar un papel importante no solo en la identificación de pacientes con alto riesgo de MetS, sino también en el manejo agresivo de la hipertensión y la dislipidemia con el fin de evitar más agresiones metabólicas.

Durante el desarrollo del proceso investigativo se observó, que el MetS es un grupo de factores de riesgo aterogénico que cada uno y en combinación aumentan proporcionalmente el riesgo de desarrollar ECV y mortalidad por ECV. Existe evidencia emergente que sugiere que el MetS es un factor de riesgo independiente para ECV y DM2. El MetS es un tema muy complejo que involucra todas las facetas del riesgo de ECV, como adiposidad abdominal, resistencia a la insulina, dislipidemia, hipertensión, trombosis, función renal, inflamación, función endotelial y muchas más. En tal sentido, la prevención y / o el manejo del MetS es un problema de salud pública crucial y, por lo tanto, los cambios en el estilo de vida que promueven una pérdida de peso moderada para quienes lo necesitan, el aumento de la actividad física y la modificación del comportamiento dietético son la piedra angular de la terapia de los Mets y sus condiciones asociadas. Algunos patrones dietéticos, como la dieta mediterránea, o el plan de dieta DASH asociado o no a la pérdida de peso corporal son las terapias nutricionales preferidas para el MetS. Los programas deben individualizarse según el patrón de vida, las preferencias y las condiciones de salud del paciente.

\section{Bibliografía}

Alberti, K., Zimmet, P., \& Shaw, J. (2005). The metabolic syndrome- a new worldwide definition. Lancet, 366.

American Heart Association. (2005). Heart Disease and Stroke Statistics: 2005 Update. Dallas, TX: American Heart Association.

Bolívar, J. (2015). Investigación Documental. México. Pax.

Castro, J. (2016). Técnicas Documentales. México. Limusa.

Davila, A. (2015). Diccionario de Términos Científicos. . Caracas: Editorial Oasis.

Kahn, R., Buse, J., Ferrannini, E., \& Stern, M. (2005). The metabolic syndrome: time for a critical appraisal. Joint statement from the American Diabetes Association and the European Association for the Study of Diabetes. Diabetologia, 1684- 1699.

Knowler, W., \& Barrett-Connor, E. (2012). Reduction in the incidence of type 2 diabetes with lifestyle intervention or metformin. N Engl J Med, 393-403.

Knowler, W., Hamman, R., \& Edelstein, S. (2015). Prevention of type 2 diabetes with troglitazone in the Diabetes Prevention Program. Diabetes , 1150- 1156 .

Lau, D., \& Dhillon, B. (2015). Adipokines: molecular links between obesity and atherosclerosis. Am J Physiol Heart Circ Physiol, H2031- H2041.

Leoncini, G., Ratto, E., Viazzi, F., \& Vaccaro, V. (2015). Metabolic syndrome is associated with early signs of organ damage in nondiabetic, hypertensive patients. J Intern Med, 454-460.

Mayo Clinic. (2019, Jun 20). Retrieved Ene 25, 2021, from https://www.mayoclinic.org/es-es/ diseases-conditions/metabolic-syndrome/symptoms-causes/syc-20351916

Miranda, P., \& DeFronzo, R. (2015). Metabolic syndrome: evaluation of pathological and therapeutic

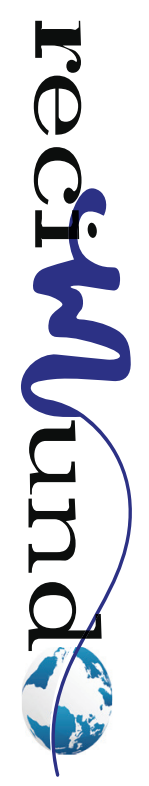


outcomes. Am Heart J , 20-32.

Pepine, C., \& Cooper-Dehoff, R. (2014). Cardiovascular therapies and risk for development of diabetes. J Am Coll Cardiol , 509-512.

Prasad, A., \& Quyyumi, A. (2014). Renin-angiotensin system and angiotensin receptor blockers in the metabolic syndrome. Circulation , 507- 512.

Reeves, M., \& Rafferty, A. (2015). Healthy lifestyle characteristics among adults in the United States. Arch Intern Med, 854-857.
Rizza, R., Vigersky, R., \& Rodbard, H. (2003). A model to determine workforce needs for endocrinologists in the United States until 2020. Diabetes Care, 1545- 1552.

Yusuf, S., \& Sleight, P. (2010). The Heart Outcomes Prevention Evaluation Study Investigators. Effects of an angiotensin-converting-enzyme inhibitor, ramipril, on cardiovascular events in high-risk patients. N Engl J Med, 342.

Zimmet, P., Alberti, K., \& Shaw, J. (2001). Global and societal implications of the diabetes epidemic. Nature , 782- 787 .

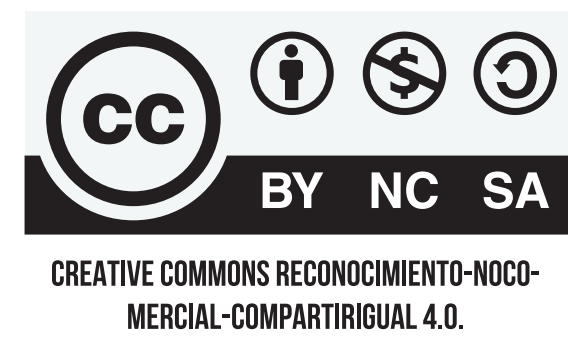

\section{CITAR ESTE ARTICULO:}

Araujo Egas, C. M., Castillo Aldás, K. M., Londoño Mateus, J. C., \& Revelo Benavides, K. M. (2021). Relación del síndrome metabólico en el riesgo de enfermedades cardiovasculares. RECIMUNDO, 5(1), 120-128. https://doi. org/10.26820/recimundo/5.(1).enero.2021.120-128 